RAIRO Operations Research

RAIRO Oper. Res. 41 (2007) 345-360

DOI: $10.1051 /$ ro:2007034

\title{
COOPERATIVE NETWORKS GAMES WITH ELASTIC DEMANDS
}

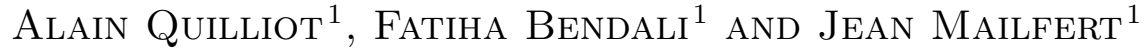

\begin{abstract}
We present here a pricing model which is an extension of the cooperative game concept and which includes a notion of elastic demand. We present some existence results as well as an algorithm, and we conclude by discussing a specific problem related to network pricing.
\end{abstract}

Keywords. Cooperative games, pricing, fixed point, network design.

Mathematics Subject Classification. 91A12, 91B24.

\section{INTRODUCTION}

Pricing problems (see $[2-4,8-10,17,26,32])$ are becoming an important topic in operations research, since deregulation processes turn pricing into a crucial issue for transport and telecommunication companies. Until recently, the gap between theoretical models derived from game theory (cooperative games $[4,8$, $10,26,31]$, non-cooperative games $[27,29]$ ), and practical applications, was really huge, essentially due to the difficulties raised by cost and demand measurement. But now, more and more powerful integrated management software turn the idea of applying game theory to practical pricing into a more realistic prospect.

Theoretical pricing models may roughly be classified according to the purpose of the pricing decision process:

- some models consider pricing as a cost imputation process or a congestion regulation tool (see [17]), and take competition into account only in

Received December 1st, 2005. Accepted November 11, 2006.

1 Laboratoire LIMOS-CNRS UMR 6158, Université de Clermont II Complexe Scientifique des Cézeaux 63177 Aubière Cedex, France;

quilliot@isima.fr, bendali@math.univ-bpclermont.fr, jean.mailfert@u-clermont1.fr

(c) EDP Sciences, ROADEF, SMAI 2007 
an implicit way (oligopoly models, cooperative models, Aumann-Shapley pricing). In the specific case of networking (with applications to the monitoring of telecommunication or transportation systems), this cost imputation process may be driven by:

- economic considerations: an operator is trying to stabilize its market share. Then, related models most often derive from the cooperative game framework, and are static aggregated models, which involve neither time nor uncertainty (see $[18,19,28]$ );

- technical considerations: pricing becomes a tool for minimizing congestion and increasing reliability. Related models often see users as players who simultaneously take routing decisions while trying to minimize some generalized cost. They usually are stochastic or dynamic models, which lead to the use of optimal control or multilevel programming techniques: see $[1,6,7,11,15,17,21,24]$;

- other models see pricing as the main parameter of any equilibrium between demand and production (Ramsay-Boiteux models, Nash equilibria, value theory, non-cooperative game models, see [20,29]). Those models are driven by the search for economic competitiveness. Some of them handle the existence or various competitors (see $[14,20]$ in the general case, and $[2,3,9,27]$ in the specific case of networking). Others express competition in an implicit way, through the expression of user's preference functions, and consider pricing as a way of adjusting production with demand (see [25]).

The reader which is interested in getting a general overview on the relation between game theory and network pricing may read the books and the surveys of Altman et al. [2], Cao et al. [8], Courcoubetis and Weber [9], Curien [10]. Still, one must be conscious that, in spite of the above mentioned technological advances, most models which aim at helping operators in taking strategic economic decisions, remain rather prospective.

We first propose here a general framework which aims at unifying both previously described points of view on pricing. Pricing is understood here according to the classical economic point of view, which means as a tool dedicated to market share segmentation. We mix the cooperative game framework, which focuses on market share stability, with the production/demand equilibrium based models, by introducing the price elasticity notion into the cooperative game framework. Next we turn to the specific case of network pricing, and we study the way it may be cast into our general framework. While pricing remains here related to economic considerations, we also take into account the fact that user's demand in relation to a transportation or a telecommunication network is elastic to various QoS (quality of service) indicators, (mean delays, expected transportation times, ...), and propose models which may yield several equilibria with distinct related activity levels. 


\section{A MIXED MODEL FOR PRICING WITH PRICE ELASTIC DEMANDS}

Notation. Let $X$ be a set. We denote by $\mathbf{P}(x)$ the set of all the subsets of $X$. We denote by 1 the $X$ indexed vector whose all coordinates are equal to 1 . If $z$ is a $X$ indexed vector and if $A$ is a subset of $X$, then we denote by $z^{A}$ the projection of $z$ on $A$, that means the $X$ indexed vector which is null for any index $x$ in $X-A$ and which coincides with $z$ for any $\mathrm{x}$ in $A$. We denote by $z_{A}$ the restriction of $z$ on $A$, which is the $A$ indexed vector which coincides with $z$ on $A$. We denote by $R$ the set of the real numbers.

\subsection{Recall: Cooperative games}

A cooperative game $J=(X, V)$ is defined by a set $X$ and by a cost function $V$ from $P(X)$ to $R$ such that:

- $V(\emptyset)=0$;

- $V$ is increasing: if $A \subset B$ then $V(A) \leq V(B)$.

For such a game $J$, the set $P(X)$ is called the coalition set.

$J$ is said to be sub-additive if, for any $A, B \in P(X)$, we have $V(A \cup B) \leq$ $V(A)+V(B)$.

The core of $\mathrm{J}$ is the set $C O(J)$ of all the price vectors $p=\left(p_{x}, x \in X\right)$ such that:

- $\sum_{x \in X} p_{x}=V(X)$

- For any $A \in P(X), \sum_{x \in A} p_{x} \leq V(A)$.

This set may be empty. In the case of a pricing problem, the core concept reflects a notion of stability.

Example: the set $\mathbf{X}$ is a customer set. Let us suppose that a telecommunication operator $O 1$ is settling an infrastructure $I$, which is likely to be used by a customer set $X$. Then a coalition $A$ may be viewed as a market share, and the cost value $V(A)$ is the cost of an infrastructure $I_{A}$, endowed with the same technical characteristics as $I$, which would be exclusively dedicated to the members of $A$ by a competitor $O 2$ of $O 1$. The eventual non-vacuity of the core will allow $O 1$ to protect itself from losing such a market share.

Remark 1. The core concept does not take into account competitors in an explicit way. In order to clearly make appear the various actors of a given market, one needs to introduce non-cooperative game models [27].

\subsection{RECALL: RAMSAY-BOITEUX MODEL FOR PRICING}

This model takes into account the fact that demands are price elastic. It considers an operator which is able to put on the market a set $I$ of goods and services. The production cost of a production vector $d=\left(d_{i}, i \in I\right)$ is a quantity $C(d)$ and the demand induced by a price vector $p=\left(p_{i}, i \in I\right)$ is a function $D(p)=\left(D_{i}(p), i \in I\right)$ of $p$. Then the RAMSAY-BOITEUX pricing process computes the prices as an inverse function of the $D_{i}$ functions. 


\subsection{A MiXED MODEL: COOPERATIVE GAMES WITH PRICE ELASTIC DEMANDS}

The price dependency of the demands, which is present in the RAMSAYBOITEUX model as well as in the non-cooperative game models, needs to be taken into account as soon as strategic decision related to market segmentation is at stake. Practically, trouble comes from the fact that modelling price elasticity is very difficult. Still, more and more powerful computing tools tend to make it easier. In order to better explain our approach, let us come back to our previous example.

Example: $\mathbf{X}$ is a customer set. Let us consider the operator $O 1$ of our previous example, and let us suppose that $X$ is a set of origin/destination pairs asking for connections. Then any connection involving such a pair $x$ will be affected with some price $p_{x}$. This price will induce a demand level $d_{x}$, which will be a fraction of all the potential connections related to $x$. This demand will depend on the whole vector $p=\left(p_{y}, y \in X\right)$, and satisfying the demand vector $d=\left(d_{y}, y \in X\right)$ will in turn induce a cost $C(d)$ for $O 1$. Then $O 1$ will have to fix $p$ in such a way that financial equilibrium is ensured and that no operator $O 2$, working with a similar technology, may propose, while limiting itself to some well-choosen subset $A$ of the customer set $X$, a cheaper service.

This leads us to introduce a general model of Cooperative Game with Elastic Demands, which tries to unify both previous models. An instance of such a cooperative game with elastic demands will be defined by a 3 -tuple $G=(X, C, D)$ in such a way that $X$ is a finite set (customers or services) and that:

- First Continuous Monotonicity Assumption: $C$ is a continuous increasing cost function such that for any production vector $d=\left(d_{x}, x \in X\right) \geq 0$, the induced cost $C(d)$ satisfies: $C(0)=0$;

- Second Continuous Monotonicity Assumption: the demand function which, to any vector price $p$, makes correspond a demand vector $D(p)=\left(D(p)_{x}\right.$, $x \in X) \geq 0$, is such that for any $x \in X$, the component function $D_{x}$ is continuous and decreasing.

Then we shall say that a pair $p=\left(p_{x}, x \in X\right) \geq 0, d=\left(d_{x}, x \in X\right) \geq 0$, is in the Core of $G$ iff:

- $d=D(p) ;$ (Price versus Demand Adequation Constraint);

- $p . d=C(d) ;$ (Financial Global Equilibrium Constraint);

- there does not exist (Stability Constraint) a subset A of X, a price vector $p^{\prime}$ and a demand vector $d^{\prime}$ such that:

$-p_{X-A}^{\prime}=p_{X-A} ; p_{A}^{\prime}<p_{A}$

$-d_{X-A}^{\prime}=0 ; d_{A}^{\prime} \geq d_{A}$

$-C\left(d^{\prime}\right)=p_{A}^{\prime} \cdot d_{A}^{\prime}$;

$-D\left(p^{\prime}\right)_{A}=d_{A}^{\prime}$.

Remark 2. The $d_{x}$ quantities must be interpreted here as fractional market shares. Due to this, the above model is a non discrete one, and null prices are eventually allowed. Also, in the case of fixed (non elastic) demands, the above model contains the usual cooperative game model. 
Remark 3. In practical applications, properly modelling the functions $C$ and $D$ will be a very hard challenge.

\section{An EXISTENCE RESUlt RELATED TO THE MIXED MODEL}

What we are going to state here is a result which will provide us with a necessary and sufficient condition for the non-vacuity of the core of a regular game. So we consider some cooperative game with elastic demands $G=(X, C, D)$.

The demand function $D$ is said to be strongly regular if it is strictly positive and if the scalar product $p \cdot D(p)$ is a strictly increasing function of $p$. The game $\mathrm{G}$ is said to be strongly regular if the function $D$ is strongly regular.

We say that this demand function $D$ is simple if for every $x \in X, D_{x}(p)$ only depends on $p_{x}$.

We say that the game $G=(X, C, D)$ is simply regular if the demand function $D$ is simple and if there exists a $X$-indexed positive price vector $\alpha=\left(\alpha_{x}, x \in X\right)>0$ such that:

- the function which, to any price vector $p$, makes correspond the product $p \cdot D(p)$, is a strictly increasing function of $p$, on the domain $\pi(\alpha)$ defined by:

$\pi(\alpha)=\left\{p=\left(p_{x}, x \in X\right)\right.$ such that for any $\left.x \in X, 0 \leq p_{x} \leq \alpha_{x}\right\}$.

- for any $x \in X, \alpha^{\{x\}} \cdot D\left(\alpha^{\{x\}}\right) \geq C\left(D(\alpha)^{\{x\}}\right)$.

We notice that if the demand function $D$ is both strongly regular and simple, then the game is simply regular.

An example: Let us suppose that, for any $x$, the function which, to a price vector $p$, makes correspond the demand $D_{x}(p)$ is piecewise linear and may be writen:

$D_{x}(p)=D_{x} \cdot\left(2 A_{x}-p_{x}\right)$ if $p_{x} \leq 2 . A_{x}$, and $D_{x}(p)=0$ else, where $A_{x}$ is a constant coefficient.

Let us also suppose that $C(d)$ is given by the formula: $C(d)=\left(\sum_{x \in X} d_{x}\right)^{1 / 2}$.

Then we see that the induced game is simply regular if for any $x$ in $X,\left(D_{x}\right)^{1 / 2}$. $\left(A_{x}\right)^{3 / 2} \leq 1$.

In order to enable us to state our main result, we are first going to prove the two following lemmas:

Lemma 3.1. Let $C$ be a cost function which satisfies the First Continuous Monotonicity Assumption (E1) and let $D$ be a demand function which satisfies the Second Continuous Monotonicity Assumption (E2). Then any element which is in the core of the game $G=(X, C, D)$ is also a solution of the following program:

Core $(G):\left\{\right.$ Compute $p=\left(p_{x}, x \in X\right) \geq 0, d=\left(d_{x}, x \in X\right) \geq 0$, such that:

- for any subset $A$ of $X, \sum_{x \in A} p_{x} \cdot d_{x} \leq C\left(d^{A}\right)$;

- $p . d=C(d)$;

- $d=D(p)\}$.

Proof. Let us check that if a pair $(p, d)$ is in the core of the game $G=(X, C, D)$, then it satisfies the constraints of the program $\operatorname{Core}(G)$. We only have to prove 
that if $A$ is some subset of $X$, then: $\sum_{x \in A} p_{x} \cdot d_{x} \leq C\left(d^{A}\right)$. Let us suppose that the converse is true and let us consider $A \subset X$ such that: $\sum_{x \in A} p_{x} . d_{x}>C\left(d^{A}\right)$. Since $C\left(d^{A}\right) \geq 0$ and since the function $C$ is monotonic, we may suppose, without any loss of generality, that $p_{x}>0$ for any $x$ in $X$. So we may consider, for any number $\tau \geq 0$, the price vector $p^{\tau}$ defined by $p_{X-A}^{\tau}=p_{X-A}$ and $p_{A}^{\tau}=\tau \cdot p_{A}$. Then there exists $\tau^{*}$ in the $[0,1]$ interval such that $p_{A}^{\tau^{*}} \cdot D\left(p^{\tau^{*}}\right)_{A}=C\left(D\left(p^{\tau^{*}}\right)^{A}\right)$. It becomes sufficient to set: $p=p^{\tau^{*}}, d_{X-A}=0$ and $d_{A}=D\left(p^{\tau^{*}}\right)_{A}$, in order to get the (E4) relationships, and to deduce that the pair $(p, d)$ cannot be in the core of $G$.

Lemma 3.2. Let $D$ some strongly regular demand function. Then the core of the cooperative game with elastic demands $G=(X, C, D)$ is exactly the solution set related to the constraints of Core $(G)$.

Proof. Lemma 3.1 tells us that any element of the core of $G$ is also a solution of the program Core $(G)$. Conversely, let $(p, d)$ a solution of $\operatorname{Core}(G)$. Let us suppose that there exists $A \subset X$ and a price vector $p$ such that: $p_{X-A}=p_{X-A}, p_{A}<p_{A}$ and $C\left(D(p)^{A}\right)=p_{A} \cdot D(p)_{A}$. We have (since $D$ is decreasing), $D(p)_{A} \geq D(p)_{A}$, and also (since $C$ is increasing) $C\left(D(p)^{A}\right) \geq C\left(D(p)^{A}\right)$. We also have (since $(p, d) \in \operatorname{Core}(G)): \sum_{x \in A} p_{x} \cdot d_{x} \leq C\left(D(p)^{A}\right)$. Then the strict monotonicity of the function which, to any price vector $q$, makes correspond the scalar product $q \cdot D(q)$, combined with the monotonicity of $D$, implies the inequality $p_{A} \cdot D(p)_{A}<p_{A} \cdot D(p)_{A}$, which induces a contradiction.

We are now able to state the main result of this section. Its intuitive meaning is that if the cost function $C$ induces some kind of scale effects, i.e, if its related marginal costs decrease in an adequate way, then the cooperative game with elastic demands $G=(X, C, D)$ has a non-empty core, whatever be the demand function $D$, provided that this function makes the game $G$ to be strongly or simply regular. Formally, we get a general Existence theorem 3.3.

Theorem 3.3. Given a finite, non empty set $X$ and a cost function $C$ which satisfies the First Continuous Monotonicity Assumption (E1). We suppose that there exists some number $\lambda \geq 0$ such that for any vector $d \geq 0, C(d) \leq \lambda \cdot N(d)$, where $N$ denotes the euclidian norm. Then the two statements (1) and (2) below are equivalent:

(1) for any demand function $D$ which satisfies the Second Continuous Monotonicity Assumption (E2), and which is such that the game $G=(X, C, D)$ is strongly or simply regular, the core of the game $G=(X, C, D)$ is not empty;

(2) for any vector $\mu=\left(\mu_{A}, A \in P(X)\right) \geq 0$, which is balanced, i.e., such that, for any $x \in X$, we have $\sum_{A}: x \in A \mu_{A}=1$, and for any vector $d=\left(d_{x}, x \in\right.$ $X) \geq 0$, we have: $C(d) \leq \sum_{A \in P(X)} \mu_{A} c \operatorname{dot} C\left(d_{A}\right)$. (E5)

Remark 4. The hypothesis involving the quotient $\frac{C(d)}{N(d)}$ comes from the usual degressivity of the marginal production costs. But it implies that no fixed initialization costs are taken into account. 
Proof. Let us first associate with $G$ and with any fixed demand vector $d$, a cooperative game $J(G, d)=\left(X, V_{G, d}\right)$, by setting: for any subset $A$ of $X$, $V_{G, d}(A)=C\left(d^{A}\right)$. Let us also recall the Bondareva-Shapley Theorem (see [5]): the core of a cooperative game $(\mathrm{X}, \mathrm{V})$ is non-empty if and only if for any balanced vector $\mu=\left(\mu_{A}, A \in P(X)\right) \geq 0$, we have: $V(X) \leq \sum_{A \in P(X)} \mu_{A} \cdot V(A)$.

Then, let us prove the implication $(1) \Rightarrow(2)$.

In order to do it, let us suppose that (2) is false. Then there exists some demand vector $d=\left(d_{x}, x \in X\right) \geq 0$ and some balanced vector $\mu=\left(\mu_{A}, A \in P(X)\right) \geq 0$, such that (E5) is not satisfied. Let $D_{x}, x \in X$ be the constant demand functions with value equal to $d_{x}$. They are both simple and strongly regular (since $d$ is strictly positive) and they satisfy (E2). If a pair $\left(p^{*}, d^{*}\right)$ is in the core of the cooperative game with elastic demands $G$, then 3.1 tells us that the price vector $q^{*}$ which is defined, for any $x$ in $X$, by: $q_{x}^{*}=p_{x}^{*} \cdot d_{x}$, must be in the core of the cooperative game $J(G, d)$. But the Bondareva-Shapley Theorem tells us that this core must be empty if (E5) is not satisfied, which means that the pair $\left(p^{*}, d^{*}\right)$ cannot exist.

Let us now prove the converse implication $(2) \Rightarrow(1)$.

We proceed in two steps.

First step: we consider only the case when the game $G=(X, C, D)$ is strongly regular. Let us first recall that a convex, compact, and upper semi-continuous point-to-set mapping from $R^{n}$ to $R^{m}$ is a function $\Gamma$ which to any $x$ in $R^{n}$ makes correspond some convex and compact subset $\Gamma(x) \subset R^{m}$ in such a way that, for any open subset $U \subset R^{m}, \Gamma^{+}(U)=x \in R^{n} \backslash \Gamma(x) \subset U$ is an open subset of $R^{n}$. Kakutani Theorem, [30], says that if $\Lambda$ is some convex compact subset of $R^{n}$, and if $\Gamma$ is some convex, compact, and upper semi-continuous point-to-set mapping from $\Lambda$ into itself, then there exists $x$ (a fixed point) in $\Lambda$ such that $x \in \Gamma(x)$.

For any unit price vector $p \geq 0$ (Bondareva Theorem [5]), the core of the cooperative game $J(G, D(p))$ is non-empty as soon as side (2) of Theorem 3.3 is true. In such a case, this core subset is a compact and convex subset of $R^{\operatorname{Card}(X)}$. We may define a convex, compact and upper semi-continuous point-to-set mapping $K$ by setting, for any price vector $p=\left(p_{x}, x \in X\right) \geq 0: K(p)=$ core of the cooperative game $J(G, D(p))$. Through composition, and taking into account the fact that the function $D$ is strictly positive (since $G$ is strongly regular), we see that the point-to-set mapping $K^{*}$ which is defined, for any unit price vector $p=\left(p_{x}, x \in X\right) \geq 0$, by: $K^{*}(p)=\left\{u=\left(u_{x}, x \in X\right) \backslash u_{x}\right.$ may be writen $u_{x}=\frac{q_{x}}{d_{x}}$ with $d_{x}=\bar{D}(p)_{x}$ and $\left.q_{x} \in K(p)\right\}$, is also convex, compact and upper semicontinuous from $R^{\operatorname{Card}(X)}$ into itself. Moreover, we also supposed the existence of a number $\lambda \geq 0$ such that for any demand vector $d \geq 0, C(d) \leq \lambda \cdot N(d), N$ being the euclidian norm. It comes that, if $p$ is some unit price vector, if $q$ is in $K(p)$ and if $x$ is in $X$, then the inequality $q_{x} \leq C\left(d^{\{x\}}\right)=C\left(D(p)^{\{x\}}\right)$ also yields $\frac{q_{x}}{d_{x}} \leq \lambda$. This means that if $u$ is in $K^{*}(p)$, then $u_{x} \leq \lambda$. We deduce that if $\Lambda$ is the set $\left\{p=\left(p_{x}, x \in X\right) \geq 0 \backslash S u p_{x \in X} p_{x} \leq \lambda\right\}$, then $K^{*}$ is a convex, compact and upper semi-continuous point-to-set mapping from $\Lambda$ into itself. It follows that there exists $p * \in \Lambda$ which is a fixed point for $K^{*}$. So we set $d^{*}=D\left(p^{*}\right)$, and we 
check that the vector $q^{*}$ which is defined, for any $x \in X$, by: $q_{x}^{*}=p_{x}^{*} \cdot d_{x}^{*}$, is in the core of the cooperative game $J\left(G, d^{*}\right)$, and that we have $1 \cdot q^{*}=C\left(d^{*}\right)$ and: $\forall A \subset X, \sum_{x \in A} p_{x}^{*} \cdot d_{x}^{*} \leq C\left(d^{* A}\right)$. Thus $p^{*}$ is a solution of the constraint set Core $(G)$. We conclude by using the fact that the game $G(X, C, D)$ is strongly regular and by applying Lemma 3.2.

Second step: we consider now the case when the game $G=(X, C, D)$ is simply regular. So, let us introduce a $X$ indexed positive price vector $\alpha=\left(\alpha_{x}, x \in X\right)>0$ such that:

- $p \cdot D(p)$ is $p$ strictly increasing, on the domain $\Pi(\alpha)=\left\{p=\left(p_{x}, x \in\right.\right.$ $\left.X) \forall A \backslash x \in X, 0 \leq p_{x} \leq \alpha_{x}\right\}$;

- for any $x \in X, \alpha^{\{x\}} \cdot D\left(\alpha^{\{x\}}\right) \geq C\left(D\left(\alpha^{\{x\}}\right)\right)$.

We may find, through the use of simple topology tricks, a demand function $D^{*}$ such that:

- $D^{*}$ is both simple and strongly regular;

- $D^{*}$ coincides with $D$ on the domain $\Pi(\alpha)$.

Because of the first step, there exists some price vector $\beta$ which is in the core of the game $G^{*}=\left(X, C, D^{*}\right)$. This vector $\beta$ is a solution of the constraint set $\operatorname{Core}\left(G^{*}\right)$. Let us set $\delta=D^{*}(\beta)$, and let us suppose that, for some $x \in X$, we have $\beta_{x}>\alpha_{x}$. Since, for any subset $A$ of $X$, we have: $\sum_{x \in A} \beta_{x} \cdot \delta_{x} \leq C\left(\delta^{A}\right)$, it also comes that if $A=\{x\}$, then: $\alpha^{\{x\}} \cdot D\left(\alpha^{\{x\}}\right)=\alpha^{\{x\}} \cdot D^{*}\left(\alpha^{\{x\}}\right)<\beta^{\{x\}} \cdot D^{*}\left(\beta^{\{x\}}\right)=$ $\beta_{x} \cdot \delta_{x} \leq C\left(\delta^{\{x\}}\right) \leq C\left(D\left(\alpha^{\{x\}}\right) \leq \alpha^{\{x\}} \cdot D\left(\alpha^{\{x\}}\right)\right.$, which also means a contradiction. Therefore, for any $x \in X$, we have $\beta_{x} \leq \alpha_{x}$. But then, we only have to apply the definitions to check that $\beta$ also belongs to the core of the game $G=(X, C, D)$. We conclude.

Remark 5 (about continuity and upper semi-continuity). As a matter of fact, assuming that both the cost function $C$ and the demand function $D$ are upper semi-continuous, is sufficient in order to get that the point-to-set mapping $K^{*}$ is upper semi-continuous, and thus to state Theorem 3.3. Still, one should be aware that, even when the function $C$ and $D$ are continuous, the above point-to-set mapping $K$ may not be continuous. Thus upper semi-continuity is necessary in order to get a correct proof of Theorem 3.3.

\section{The CAse of A Network Design Game}

We are now going to adapt the above model to the case of a specific network design problem, according to which demands depends not only on prices but also on the quality of service (QoS) of the routing process. So we consider here a strongly connected communication or transportation network $H=(Z, E)$, where $Z$ is a set of nodes and $E$ is a set of arcs, as well as a specific arc set $U \subset E$, which is likely to support a specific high QoS (speed) transportation mobile infrastructure (for instance a shuttle fleet) $F$. We suppose that any $\operatorname{arc} e$ in $E$ is endowed with 
a financial cost $c_{e}$ and with a routing or quality (QoS) cost $t_{e}$, in such a way that this cost $t_{e}$ is significatively smaller if $e \in U$ than if $e \notin U$.

The customer set $X$ is defined here by a family of origin/destination pairs of vertices $\left(o_{x}, s_{x}\right), x \in X$. The demand $d_{x}$ of any user group $x$ in $X$ in relation to the infrastructure $F$ may involve the transportation of passengers, goods, luggages... It is supposed to depend on both the unit price $p_{x}$ which is charged to the user group $x$ for the access to $F$, and on the routing QoS (Quality of Service) of the induced connection.

QoS and Financial Reference Values: for every $x \in X$, we denote by $T o p_{x}$ the length of a $t$ shortest path $\Gamma_{x}$ from $o_{x}$ to $d_{x}$, (computed for the QoS costs $t_{e}, e \in E$ ), and we denote by $Q r e f_{x}$ the length of a $c$ shortest circuit (computed for the financial costs $c_{e}, e \in E$ ), which contains the shortest path $\Gamma_{x}$. We respectively call $T o p_{x}$ and $Q r e f_{x}$ the $Q o S$ and Financial Reference Values associated with the customer $x \in X$.

Infrastructure operator and customer decisions: let us suppose that coefficients $t_{e}, e \in E$, are time coefficients. Then, taking an infrastructure decision consists, for a given operator who manages a shuttle fleet, in determining routes and trafic volumes. This decision might be summarized as some infrastructure flow $F$, with support on the arc set $U$. Designing and pricing $F$ will induce, for any $x \in X$, some running time (QoS level) $T_{x}$, and, consequently, some demand level $d_{x}$ as well as some routing decision which might be summarized by some flow $f(x)$. So, infrastructure and pricing decisions should be simultaneously taken, while meeting the induced demands and ensuring a financial equilibrium. But, if we suppose that demand levels $d_{x}$ and QoS levels $T_{x}, x \in X$, are already known, the operator only must run its fleet under the smallest possible costs, while ensuring, for any user $x \in X$, to go from origin $o_{x}$ to destination $s_{x}$ in no more than $T_{x}$ time units. In such a case, this operator should solve the following program $\operatorname{NETWORK}(d, T)$ :

Linear program NETWORK $(d, T):\{$ Find a flow $F \geq 0$ and a multiflow $f=f(x), x \in X$, both defined on $H$, such that :

- the flow $F$ represents the mobile infrastructure decision of the operator (the mean trafic of the shuttles), and only involves $\operatorname{arcs}$ of $U$ : for any $e \notin U, F_{e}=0$;

- every flow $f(x)$ represents the routing of the demand (passengers, goods, luggages...) $d_{x}$ from $o_{x}$ to $s_{x}$, and is submitted to the following QoS constraint: $d_{x} \cdot T_{x} \geq t \cdot f(x)$;

- $F$ and $f$ are tied by a capacity constraint on the $\operatorname{arcs}$ of $U$ : for any $e \in U, F_{e} \geq \sum x \in X f(x)_{e} ;(\underline{\text { Comment: }}$ this constraint is an inequality, since the "shuttle" of F may not be fully loaded). and which minimize the quantity $c \cdot F\}$.

We denote by $W(d, T)$ the optimal value of this program and we call it the cost induced by the pair $(d, T)$.

The Network Design Multicriterion Game MG-NET.

Since infrastructure and price decisions should be simultaneously taken, we are 
led to define the Network Design Multicriterion Game MG-NET $=(H, X, D=$ $\left(D_{x}, x \in X\right)$ ), by considering that the access demands $d_{x}, x \in X$, depend on both the unit access prices $p_{x}, x \in X$, and on the routing QoS $\operatorname{costs} T_{x}=\frac{(t . f(x))}{d_{x}}, x \in X$, in such a way that, for any $x \in X, d_{x}$ may be written $d_{x}=D_{x}\left(p_{x}, T_{x}\right)$, where $D_{x}$ is a continuous and decreasing positive or null function from $\Re^{2}$ to $\Re$.

Our model of Core of a Cooperative Game with Elastic Demands may be extended by telling that a 3-uple $p=\left(p_{x}, x \in X\right) \geq 0, T=\left(T_{x}, x \in X\right) \geq 0, d=$ $\left(d_{x}, x \in X\right) \geq 0$, is in the Core of the Network Design Multicriterion Game MGNET defined by the program $\operatorname{NETWORK}(d, T)$ and by the demand functions $\left(D_{x}, x \in X\right)$, iff:

- $d=D(p, T)$;

- there exists an optimal solution $(F, f)$ of the NETWORK(d,T) program which is such that : $c \cdot F=p \cdot d$;

- there does not exist $A \subset X$ and any 3 -uple $(p, T, d)$ (price, routing cost, demand) such that:

$$
\begin{aligned}
& -(p, T)_{A}<(p, T)_{A} ; d_{A} \geq d_{A} ; \\
& -p_{X-A}=d_{X-A}=0 ; T_{X-A}=0 \\
& -d_{A}=D(p, T)_{A} ; \\
& -W(d, T)=\text { optimal value of the program } \operatorname{NETWORK}(d, T)=d \cdot p .
\end{aligned}
$$

One interprets this model by refering to our shuttle operator: if he prices the access to his fleet according to an element of the core of the game MG-NET, then no other operator operating under similar costs and technologies, can identify some market share inside the customer set $X$ and make him a better offer.

Remark 6. Defining a game while using a cost function associated with some linear program, is characteristic of cooperative game modelling [18,19,31]. Innovation comes here from the fact that we take into account price/QoS elastic demands and (economical costs)/(QoS level) tradeoff configurations.

Generalized Costs and the Regularity property.

For any value $k \geq 0$, we set: $Q_{k, x}\left(p_{x}, T_{x}\right)=\left(p_{x}+k \cdot\left(T_{x}-T_{o} p_{x}\right)\right) \cdot D_{x}\left(p_{x}, T_{x}\right)=$ generalized cost induced by the generalized cost parameter $k$ and by the routing of the demand $D_{x}\left(p_{x}, T_{x}\right)$ under price/QoS pair $\left(p_{x}, T_{x}\right)$. Doing this leads us to define, for any demand vector $d$ and any value $k$, the following program $\operatorname{NET}(d, k)$ :

$\operatorname{NET}(d, k):\{$ Find a flow $F \geq 0$ and a multiflow $f=f(x), x \in X$, both defined on $H$, such that:

- every flow $f(x)$ represents the routing of the demand $d_{x}$ from $o_{x}$ to $s_{x}$;

- for any $e$ in $U, F e \geq \sum_{x \in X} f(x)_{e}$;

- for any $e \notin U, F_{e}=0$;

and which minimizes the quantity $\left.c \cdot F+k \cdot t \cdot\left(\sum_{x \in X} f(x)\right)\right\}$.

We set $V(d, k)=$ optimal value of the program $\operatorname{NET}(d, k)$. For any coalition $A \subset X$, we set $V(d, k)^{A}=$ optimal value of the program $N E T(d, k)^{A}$ which is the restriction of $\operatorname{NET}(d, k)$ to the variables and constraints which are related to $A$. Doing this allows us to define a cooperative game COOPGAME-NET $(d, k)=$ $(X, V(d, k))$. 
Remark 7. The above generalized cost parameter $k$ is here a value-per-time scaling coefficient which mixes financial costs and QoS costs into a unique global generalized cost.

We say that our game MG-NET is strongly regular if any function $D_{x}$ is strictly positive and if for any value $k \geq 0$, the generalized cost $\left(p_{x}+k \cdot\left(T_{x}-T_{o p}\right)\right)$. $D_{x}\left(p_{x}, T_{x}\right)$ is a strictly increasing function of the pair $\left(p_{x}, T_{x}\right)$.

We say that this game is regular if there exists a value $k>0$, a $X$-indexed positive price vector $\alpha=\left(\alpha_{x}, x \in X\right)>0$, and a $X$-indexed positive routing cost vector $\delta=\left(\delta_{x}, x \in X\right)>0$, such that:

- for any $x \in X$, the generalized cost $\left(p_{x}+k \cdot\left(T_{x}-T o p_{x}\right)\right) \cdot D_{x}\left(p_{x}, T_{x}\right)$ is a strictly increasing function of the pair $\left(p_{x}, T_{x}\right)$, on the domain $\Pi\left(\alpha_{x}, \delta_{x}\right)$ which is defined by $0 \leq p_{x} \leq \alpha_{x}$ and $0 \leq T_{x}-T o p_{x} \leq \delta_{x}$;

- for any $x \in X$, both inequalities $Q r e f_{x} \leq \alpha_{x}$ and $Q r e f_{x} \leq k \cdot \delta_{x}$, which involve the financial reference $Q r e f_{x}$ value associated with $x$, are true. (E6)

Of course, if our game is strongly regular, it is also regular.

\section{A Theoretical Result.}

We are now going to prove that if the above game MG-NET $=(H, X, D)$ is regular, then its core is non empty.

Lemma 4.1. For any strictly positive value of $k$, the function $V$ which, with any demand vector $d$, associates the value $V(d, k)$, is continuous and increasing. Besides, there exists $\lambda \geq 0$ such that for any demand vector $d \geq 0$, we have $V(d, k) \leq \lambda \cdot N(d)$, where $N$ is the euclidian norm.

Proof. The fact that $V$ is continuous and increasing is obvious. In order to get the part of the lemma which is related to the coefficient $\lambda$, we consider the polyedron $\Pi^{*}$ defined by the dual program of $\operatorname{NET}(d, k)$ and we denote by $S$ the vertex set of $\Pi^{*}$. Let us denote by $d^{*}$ the bound vector of the program $\operatorname{NET}(d, k)$. A part of this vector is indexed on the arc set $E$ and is null. Another part is indexed on the vertex pairs $(y, x)$, that means on the constraints which express that the flow $f(x)$ satisfies the Kirshoff Law in every vertex $y$ of the network $H$. Since the program $\operatorname{NET}(d, k)$ is bounded, any value $V(d, k)$ may be written (duality) $V(d, k)=\operatorname{Sup}_{s \in S}\left\{s \cdot d^{*}\right\}$. We notice that this bound vector $d^{*}$ is the image of $d$ through some linear function and we conclude.

In order to keep on toward our main result, let us consider now a pair $(F, f)$ which is an optimal value of the program $\operatorname{NET}(d, k)$, and let us suppose that the part of the bound vector $d^{*}$ of $\operatorname{NET}(d, k)$ which expresses that the flow $f(x)$ satisfies the Kirshoff Law in every vertex $y$ of the network $H$, is indexed as follows: $d^{*}=\left(d_{y, x}^{*}, y, x \in Z\right)$. We also may suppose that $d^{*}$ is such that, for any $x \in Z$ : $d_{o_{x}, x}^{*}=d_{x}, d_{s_{x}, x}^{*}=-d_{x}$, and $d_{y, x}^{*}=0$ if $y$ is different from $s_{x}$ and $o_{x}$. Then we suppose that $d$ is strictly positive and we set, for any $x \in X: T_{x}=\frac{(t . f(x))}{d_{x}}=$ QoS of $f$ for user $x$. We say that $T=\left(T_{x}, x \in X\right)$ is the QoS Vector associated with $f$. Doing it allows to get: 
Lemma 4.2. Let $u=\left(u_{z}, z \in Z\right), v=\left(v_{z, x}, z \in Z, x \in X\right), w=\left(w_{e}, e \in A\right) \geq 0$ be some optimal solution of the dual program $N E T^{*}$ of the program $N E T(d, k)$. We suppose $d>0$. Then for any $x \in X$, we have $k \cdot T_{x} \leq d_{x} \cdot\left(v_{o_{x}, x} v_{s_{x}, x}\right)$.

Proof. The vector $v$ may be choosen in such a way that for any $x, v_{s_{x}, x}=0$. Let us consider $x_{o} \in X$, and let us consider some positive number $\delta<d_{x_{o}}$. We know that $v_{o_{x} o}, x_{o} \cdot \delta \geq V(d, k)-V(d, k)$, where $d$ is obtained from $d$ by substracting $d$ from $d_{x} o$ (subdifferentiality of the dual solution of the linear program $\mathrm{NET}(d, k)$ ). We get a feasible solution $f$ of $\operatorname{NET}(d, k)$ by removing some amount $d$ from the flow vector $f(x o)$, in such a way that the product $k \cdot t \cdot\left(\sum_{x \in X} f(x) k \cdot t \cdot\left(\sum_{x \in X} f(x)\right)\right.$ is equal to $k \cdot \delta \cdot T_{x o}$. We deduce that $V(d, k) \leq V(d, k) k \cdot \delta \cdot T_{x} o$ and the result.

Lemma 4.3. Given some optimal solution $(F, f)$ of the linear program $N E T(d, k)$, where $d$ is supposed to be strictly positive, and $T=\left(T_{x}=\frac{t \cdot f(x)}{d_{x}}, x \in X\right)$ the related QoS Vector. Then there exists some price vector $p \geq 0$, such that the vector $q=\left(\left(p_{x}+k \cdot d_{x} \cdot T_{x}\right), x \in X\right)$ is in the core of the cooperative game COOPGAME$\operatorname{NET}(d, k)$.

Proof. Let us consider, as in Lemma 4.2, $u=\left(u_{z}, z \in Z\right), v=\left(v_{z, x}, z \in\right.$ $Z, x \in X), w=(w e, e \in A) \geq 0$, which define some optimal solution of the dual program $\mathrm{NET}^{*}$ of the program $\operatorname{NET}(d, k)$. Then we may set, for any $x$ in $X$, $p_{x}=v^{x} \cdot 1^{* x} k \cdot T_{x}$ where $1^{* x}$ is the $Z$ indexed vector which is equal to 1 in $o_{x}$, to 1 in $s_{x}$ and 0 elsewhere. Lemma 4.2 tells us that $p=\left(p_{x}, x \in X\right)$ is $\geq 0$. If $A \subset X$ represents some coalition, then we see that the restrictions of $u, v$ and $w$ to the constraints of the program $\operatorname{NET}(d, k)$ which only involve the elements of $A$, define a feasible solution of the dual program of the restriction program $\mathrm{NET}(d, k)^{A}$. We deduce the following relations $(\mathrm{E} 7)$ :

- $\sum_{x \in A}\left(p_{x}+k \cdot d_{x} \cdot T_{x}\right) \leq$ optimal Value $V(d, k)^{A}$ of the program $N E T(d, k)^{A}$;

$-\sum_{x \in X}\left(p_{x}+k \cdot d_{x} \cdot T_{x}\right)=$ optimal Value $\mathrm{V}(d, k)$ of the program $\operatorname{NET}(d, k)$.

But (E7) means that $p+k . d . T$ is in the core of the cooperative game COOPGAME$\operatorname{NET}(d, k)$.

Stating our main existence result comes now as follows:

Theorem 4.4. If the above network game $M G-N E T=(H, X, D)$ is regular, then its core is non empty.

Proof. For any strictly positive demand vector $d$ and any value $k \geq 0$ of the generalized cost parameter, let us denote by $\operatorname{Core}(d, k)$ the set of the pairs $(p, T)$, $p \geq 0, T \geq T o p$, which are such that there exists some vector $q$ in the core of the cooperative game COOPGAME-NET $(d, k)$ and some optimal solution $(F, f)$ of the linear program $\operatorname{NET}(d, k)$ which may be written: $q=\left(\left(p_{x}+k \cdot d_{x} \cdot T_{x}\right), x \in X\right)$, where $T=\left(T_{x}=t \cdot f(x) / d_{x}, x \in X\right)$ is the QoS vector related to $f$. Then, the end of the proof of Theorem 4.4 comes as a mere copy of the proof of theorem 3.3.

We first consider (step 1) the case when our game is strongly regular. Then, while $k$ remains fixed, we consider the domain $\Lambda$ defined by: $\Lambda=\{p \geq 0, T \geq$ 
Top, such that $\left.\operatorname{Sup}_{x \in X}\left(p_{x}+k \cdot d_{x} \cdot T_{x}\right) \leq \Lambda\right\}$, and the point-to-set mapping $G$ which to any pair $(p, T) \in \Lambda$ makes correspond $\Gamma(p, T)=\operatorname{Core}(D(p, T), k)$. We check that any pair $(p, T)$ which is a fixed point for this point-to-set mapping, is also in the core of the network design multicriterion game MG-NET defined by the program NETWORK $(\mathrm{d}, \mathrm{T})$ and by the demand functions $\left(D_{x}, x \in X\right)$. We conclude through application to $G$ of the Tychonof-Kakutani Fixed Point Theorem.

Next (Step 2), we consider the general case, when the game MG-NET is regular, and we pick up a value of the generalized cost parameter $k$, a $X$-indexed positive price vector $\alpha=\left(\alpha_{x}, x \in X\right)>0$, and a $X$-indexed positive routing cost vector $\delta=\left(\delta_{x}, x \in X\right)>0$, such that:

- for any $x \in X$, the generalized cost $\left(p_{x}+k \cdot\left(T_{x}-T o p_{x}\right)\right) \cdot D_{x}\left(p_{x}, T_{x}\right)$ is a strictly increasing function of the pair $\left(p_{x}, T_{x}\right)$, on the domain $\Pi\left(\alpha_{x}, \delta_{x}\right)$ which is defined by $0 \leq p_{x} \leq \alpha_{x}$ and $0 \leq T_{x}-T o p_{x} \leq \delta_{x}$;

- for any $x \in X$, both inequalities $Q r e f_{x} \leq \alpha_{x}$ and Qre $f_{x} \leq k . d_{x}$, which involve the financial reference $Q r e f_{x}$ value associated with $x$, are true. (E6bis)

A consequence of the regularity relations (E6) and (E6bis) is that (E8):

- for any $x \in X$, and for any value $u$ in $\left[0, \delta_{x}\right]$ :

$\left(\alpha_{x}+k \cdot u\right) \cdot D_{x}\left(\alpha_{x}, u+T_{o p}\right) \geq V\left(\left(0, . ., D_{x}\left(\alpha_{x}, u+T_{o p}\right), 0, . ., 0\right), k\right)-$ $k . T o p_{x} . D_{x}\left(\alpha_{x}, u+\operatorname{Top}_{x}\right)$;

- for any $x \in X$, and for any value $v$ in $\left[0, \alpha_{x}\right]$ : $\left.\left(v+k . \delta_{x}\right) \cdot D_{x}\left(v, \delta_{x}+T o p_{x}\right) \geq V\left(\left(0, . ., D_{x}\left(v, \delta_{x}+T o p_{x}\right)\right), 0, . ., 0\right), k\right)-$ $k \cdot T o p_{x} . D_{x}\left(\delta_{x}, u+\right.$ Top $\left._{x}\right)$;

Then we build demand functions $D_{x}^{*}, x \in X$, which are such that:

- the induced game is strongly regular;

- for any $x \in X$, the function $D_{x}$ coincides with $D_{x}^{*}$ on the domain $\Pi\left(\alpha_{x}, \delta_{x}\right)$.

Step 1 allows us to assert the existence of a pair $(\beta, \Delta)$ which is in the core of the game $M G-N E T^{*}=\left(H, X, D^{*}\right)$. We conclude by using (E8) and the same trick as at the end of the proof of theorem 3.3, in order to check that every pair $\left(b_{x}, D_{x}\right)$ must be in the domain $\Pi\left(\alpha_{x}, \delta_{x}\right)$, and by deducing that $(\beta, \Delta)$ must also be in the core of the game MG-NET.

Corollary 4.5. A computing scheme CORE-NETWORK

In case the game MG-NET is regular, an element of its core may be obtained by picking up a convenient value of the generalized cost parameter $k$, and by solving the following constraint system CORE-NETWORK(k):

$\{$ Find $p, T, d \geq 0$, a primal (optimal) solution $(F, f)$ and a related dual solution $u=\left(u_{z}, z \in Z\right), v=\left(v_{z, x}, z \in Z, x \in X\right), w=(w e, e \in A) \geq 0$, of the $N E T(d, k)$ program, such that:

- $d=D(p, T)$;

- for any $x$ in $X, d_{x} \cdot T_{x}=t . f(x)$; (E9) 
- for any $x$ in $X, p_{x}=v^{x} \cdot 1^{*} x k \cdot T_{x}$, where $1_{z}^{* x}=1$ if $z=o_{x}, 1$ if $z=s_{x}$ and 0 else $\}$. (E10)

Proof. Lemma 4.2 allows us to reinforce the point-to-set mapping G of the proof of Theorem 4.4, by setting:

- Core $^{*}(d, k)=\{(p, T) \geq 0$ which are such that there exists a primal solution $(F, f)$ and a dual solution $u=\left(u_{z}, z \in Z\right), v=\left(v_{z, x}, z \in Z, x \in X\right)$, $w=\left(w_{e}, e \in A\right) \geq 0$ of $\operatorname{NET}(d, k)$ such that:

$-T=\left(T_{x}=\frac{t . f(x)}{d_{x}}, x \in X\right)$;

- for any $x$ in $X, p_{x}=v^{x} \cdot 1^{* x} k \cdot T_{x}$ with $1_{z}^{* x}=1$ if $z=o_{x}, 1$ if $z=s_{x}$ and 0 else;

- $\Gamma^{*}(d, k)=\operatorname{Core}^{*}(D(p, T), k)$. We then apply to $\Gamma^{*}$ the same fixed point argument as in the proof of Theorem 4.4. If the 5 -uple $(p, T, d, F, f)$ is a solution of CORE-NETWORK $(\mathrm{k})$, then the pair $(p, T)$ is in the core of MG-NET.

We conclude.

We may derive an algorithm CORE-NETWORK from the above proof, which aims at computing an element of the core of the game MG-NET in case such an element exists. This algorithm works in a heuristic way, according to an iterative fixed point process. At any iteration of its internal loop (Stop1 loop), it starts from some current pair $(p, T)$, next computes the induced demand $d$ and solves the linear program $\operatorname{NET}(d, k)$, and finally modify $(p, T)$ in order to reduce the gap between $(p, T)$ and the price/QoS pair which results from the application of the formulae (E9), (E10) to the primal solution $(F, f)$ and to the dual solution $(u, v, w)$ of $\operatorname{NET}(d, k)$.

\section{CORE-NETWORK Algorithm;}

Provide the generalized cost parameter $k$ with some value; Not Stop; While Not Stop do

(*main loop: we try several values of $k$ until success or stop ${ }^{*}$ )

Initialize $p>0$ and $T \geq T o p$; Not Stop1;

While Not Stop1 do

( ${ }^{*}$ Internal loop: the heuristic fixed point scheme which aims at solving the CORE-NETWORK $(k)$ constraint system*)

compute $d=D(p, T)$;

Compute a primal solution $(F, f)$ and a related dual solution $u=\left(u_{z}, z \in Z\right), v=\left(v_{z, x}, z \in Z, x \in X\right), w=\left(w_{e}, e \in A\right) \geq 0$, of the $\operatorname{NET}(d, k)$ program;

For any $x$ in $X$, compute $T_{x}^{\prime}$ in such a way that $d_{x} \cdot T_{x}^{\prime}=t . f(x)$;

For any $x$ in $X$, set $p_{x}^{\prime}=v_{x} \cdot 1^{* x} k \cdot T_{x}^{\prime}$, where $1^{* x}$ is the

$Z$ indexed vector which is equal to 1 in $o_{x}$, to 1 in $s_{x}$ and 0 elsewhere;

If the relative error between $(p, T)$ and $\left(p^{\prime}, T^{\prime}\right)$ is small enough then Stop1;

Stop;

Else 
Replace $(p, T)$ by $\left(p+p^{\prime}, T+T^{\prime}\right) / 2$; Adjust Stop1;

If Stop1 then modify the value of $k$ and adjust Stop.

Remark 8. The generalized cost parameter $k$ is a key player inside the above scheme. Choosing its value will impact both the convergence of the CORENETWORK process and the quality of the resulting decision $(F, f)$.

\section{Conclusion}

We proposed here a model which could help us in understanding pricing mechanisms and in dealing with related decision problems. This model involves the cooperative game framework and tries to take into account the way users are likely to react to tariff policies. It could eventually help in conveniently pricing and designing public transportation systems in order to promote public transportation modes. Still, one must keep in mind that pricing remains a very hard issue, mainly due to essential difficulties related to cost and demand measurement.

\section{REFERENCES}

[1] E. Altman and A Markov, game approach for optimal routing in a queing network, in Annal. of Dynamic Games, Stochastic and Differential Games, Theory and Numeric Methods 5, Birkauser, Berlin (1999) 359-376.

[2] E. Altman, T. Boulogne, R. El Azouzi, T. Jimenez and L. Wynter, A survey on networking games in telecommunications. Comput. Oper. Res. (2004) 47 p.

[3] E. Altman and L. Wynter, Equilibrium, games and pricing in transportation and telecommunication networks, in Networks and Spatial Economics: special issue on crossovers between transportation and telecommunication modelling, edited by L. Altman and L. Wynter (2004) $7-21$.

[4] C.G. BIRD, On cost allocation on a spanning tree: a game approach. Networks 6 (1976) $335-350$.

[5] O.N. Bondareva, Some applications of linear programming methods to the theory of cooperative games. Problemy Kibernetica 10 (1963) 119-139.

[6] T. Boulogne, E. Altman, H. Kameda and O. Pourtallier, Mixed equilibrium in multiclass routing games. IEEE Trans. Aut. Cont. 47 (2002) 903-916.

[7] T. Boulogne, E. Altman and O. Pourtallier, Mixed equilibrium in multiclass routing games, Ann. Oper. Res. 109 (2002) 279-291.

[8] X.R. Cao, H.X. Shen, R. Milito and P. Wirth, Internet pricing with a game theoretical approach: concepts and examples. IEEE/ACM Transactions on Networking 10 (2002) 208215.

[9] C. Courcoubetis and F. Weber, Pricing communication networks-economics, technology and modelling, Wiley, New York (2003).

[10] N. Curien, Cost allocation and pricing policy: the case of french telecommunications, in Cost Allocation: Methods, Principles, Applications, edited by H.P. Young, Chap. 9, Elsevier Sciences (1985) 167-178.

[11] L.A. Da Silva, Pricing of QoS enabled networks: a survey. IEEE Com. Surv. Tutorials 3 (2000).

[12] P. Dubey and L.S. Shapley, Totally balanced games arising from programming problems. Math. Program. 29 (1984) 245-267. 
[13] D. Granot and G. Huberman, On the core and nucleolus of minimum spanning tree games. Math. Program. 29 (1984) 323-347.

[14] H.A. Eiselt, G. Laporte and J.F. Thisse, Competitive location models: a framework and bibliography. Transp. Sci. 27 (1993) 44-54.

[15] R. El Azouzi and E. Altman, Constrained traffic equilibrium in routing. IEEE Trans. Aut. Cont. 48 (2003) 1656-1660.

[16] R. El Azouzi, E. Altman and L. Wynter, Telecommunication network equilibrium with price and quality of service characteristics, in Proc. of Internat. Conf. on Teletraffic ITC, Berlin (2003).

[17] R.P. Gibbens and F.P. Kelly, Resource pricing and the evolution of congestion control. Automatica 35 (1999) 1969-1985.

[18] D. Granot and F. Granot, On some network flow games. Math. Op. Res. 17 (1992) 792-841.

[19] D. Granot and M. Maschler, Spanning network games. Int. J. Game Theor. 27 (1998) $467-500$.

[20] A. Haurie and P. Marcotte, On the relationship between Nash Cournot equilibrium and Wardrop equilibria. Networks 15 (1985) 295-308.

[21] M. Haviv, The Aumann-Shapley pricing mechanism for allocating congestion costs. Oper. Res. Lett. 29 211-215 (2001).

[22] G. Huberman, The nucleolus and the essential coalitions, in Analysis and Optimization Systems. Springer Berlin (1980) 416-422.

[23] E. Kalai and E. Zemel, Totally balanced games and flows. Math. Oper. Res. 7 (1982) 476478.

[24] F.P. Kelly, Charging and rate control for elastic traffic. Eur. Trans. Telecom. 8 (1997).

[25] Y.A. Korilis, A.A. Lazar and A. Orda, Capacity allocation under non cooperative routing. IEEE Trans. Aut. Cont. 42 (1997) 309-325.

[26] R.J. La, and V. Anantharam, Network pricing using game theoretical approach, in Proc. 38th IEEE Conf. Decision and Control 4 (1999) 4002-4007.

[27] P.J. Lederer, A competitive network design problem with pricing. Transp. Sci. 27 (1993) $25-38$.

[28] N. Meggido, Cost allocation for Steiner trees. Networks 8 (1978).

[29] J. Nash, Non cooperative games. Ann. Maths 54 (1951) 286-295.

[30] D.R. SMART, Fixed Point Theorems. Cambridge Univ Press, Cambridge tracts in Math. 66 (1974).

[31] A. Tamir, On the core of network synthesis games. Math. Program. 50 (1991) 123-135.

[32] A. Van Den Nouweland, P. Borm and W. Van Golstein, A game theoretic approach to problems in telecommunications. Manage. Sci. 42 (1996) 294-303. 